\title{
Challenges of E-Journal Club: A Case Study
}

\author{
Nazila Zarghi ${ }^{1,2^{*}}$, Seyed Reza Mazlom ${ }^{1}$, Majid Rahban ${ }^{3 \#}$ \\ ${ }^{1}$ Department of Nursing, School of Nursing and Midwifery, Mashhad University of Medical Sciences, \\ Mashhad, Iran \\ ${ }^{2}$ Education Development Center (EDC), Mashhad University of Medical Sciences, Mashhad, Iran \\ ${ }^{3}$ Department of IT, School of Nursing and Midwifery Branch, \\ Mashhad University of Medical Sciences, Mashhad, Iran \\ Email: "zarghin@mums.ac.ir, mazlomr@mums.ac.ir, Rahbanm1@mums.ac.ir
}

Received August 14 $4^{\text {th }}, 2012$; revised September 18 ${ }^{\text {th }}, 2012$; accepted September $30^{\text {th }}, 2012$

\begin{abstract}
Background: Journal clubs, founded by Sir William Osler in 1875, have been used as a teaching format in academic medicine for over a hundred years. New generation of journal club include EBM format, Audio journal club and the latest, E-journal clubs. This study aimed the challenges of helding E-journal club in MUMS. Method: For this, a special virtual space was designed for e-journal club and a practical article was selected for on-line comments. It was possible for participants to register in a 7-day time span. Leader of journal club sent an e-mail for participants and explained the process for them and asked them to read the article and leave a comment. As there was impossible for being on-line at the same time for participants, it was decided to design the second space for leaving comments. Both participants and leader could access the others' comments, so the interaction became possible for them. The time for leaving comment was considered 10 days. Result: Although participants included faculty members and students who were familiar with e-methods, there was no comment on the space. Conclusion: It seems, other involving factors should be considered. The other factors apart from educationals, influenced on performing and participating in new generation journal clubs which demands further observation.
\end{abstract}

Keywords: Electronic Journal Club; Journal Club; E-Journal Club

\section{Introduction}

Journal club has been defined as "A group of individuals who meet regularly to discuss critically the clinical applicability of articles in the current medical journals" (Linzer, 1987). Based on documents, the first recorded journal club was run at Mc Gill University in 1885, by Sir William Osler aimed to distribute and pay for journals to which he could not afford to subscribe. Nowadays, the aims have changed, but essentially the practice remains as an excellent way to remain up to date with developments in each field. In recent years, the importance of "evidence based healthcare" has grown and there has been an inclination to integrate practical evidences into clinical setting (NHS, 2010). It seems obvious that running journal club could be a key method for reviewing literature and discussing its clinical implications. The aims of any journal club should be critical evaluation of literature and assess the-state-of-the-art advances for each specialty (Gibbons, 2002). The aims and objectives of journal club include:

- To provide a bridge between research and practice and foster an application of research to the clinical setting;

- To locate the best evidence on which to base practice;

- To help research awareness;

- To keep abreast with current research;

- To strengthen critical appraisal skills;

- To understand bio statistical and epidemiological methods;

- To increase confidence in dealing with evidence/research;

- To improve professional reading habits;

*Corresponding author.

"BSc of Medical Library and Information Sciences.
- To develop multidisciplinary relationships;

- To fulfill requirements for Continual Professional Development;

- To maintain and improve professional knowledge and competence which influence on quality of care;

- To develop local guidelines (Lindquist, Robert, \& Treat, 1990).

The structure of successful journal clubs is different. Healthbased journal clubs have been in place for a long time. Participants meet regularly to critique research papers, improve their understanding of research design, biostatistics and critical appraisal. However, there is no standard process of conducting an effective journal club. The main characteristics of successful journal clubs include regular and anticipated meetings, mandatory attendance, clear long- and short-term purpose, appropriate meeting timing and incentives, a trained journal club leader to choose papers and lead discussion, circulating papers prior to the meeting, using the internet for wider dissemination and data storage, using established critical appraisal processes and summarizing journal club findings (Eval, Deenadayalan, GrimmerSomers, Prior, \& Kumar, 2008). Face to face journal clubs have been used as an atmosphere to share knowledge and discuss research findings relevant to clinical practice. However, attendance at journal club meetings has always been identified as a barrier to successful and sustainable clubs. One of the possible solutions to this is the establishment of electronic journal clubs. The on-line journal club is a useful example of the educational advantages created by the combination of technology with traditional journal club methods (Lizarondo, Kumar, \& GrimmerSomers, 2010). It not only provides an opportunity for asyn- 
chronous discussion but also allows members to participate in discussion at a time and place of convenience. To study the challenges of E-journal club, it was evaluated goal and objectives of journal club by which the challenges will be discovered through level of achievement.

The purpose of this study is to describe a teaching strategy that can be used for large numbers of participants, which improve critical appraisal skills for applying in clinical practice through participation in a university-based journal club. It aimed to provide a virtual space for journal club and study the challenges of E-journal club in Mashhad University of Medical Sciences.

\section{Methods}

For performing this study and evaluate goals and objectives, a special virtual space was designed for e-journal club, and then a paper was selected to discuss via electronic approach (Figures 1 and 2). Participants were given a 7-day time span for registration. An e-mail was for them to explain the process of E-journal club and asked them to read the paper and leave their comments on the space where was designed for this purpose. As there was impossible for participants to be on-line at the same time, it was decided to design the second space for leaving comments. Both participants and leader could access the others' comments, so they could actively interact with each other by their comments. They had 10 days for making comment. The final step was summing up the comments and making hand out for participants. An evaluation form was designed to observe the educational impacts at the end of session both for appraisal skills and knowledge of the related area.

The following criteria were considered for conducting this study based on journal club goal and objectives:

As CPR (cardiopulmonary resuscitation is one of the most critical and basic skills among different health care professions in clinical setting, it was decided to select a paper on this topic by which the findings could be applied in practice and update health care professions with current high level of evidence research (Level of Evidence A). The paper was selected from AHA (American Heart Association) which releases and updates international guidelines for CPR every five years. It was about BLS (Basic Life Support) guideline which was updated in 2010. To help participants' research awareness and strengthen critical appraisal skills, understand bio statistical and epidemiological methods and increase their confidence in dealing with evidence/ research, a critical appraisal tool was sent with paper by which a standard and strict approach was introduced to participants both for critique and dealing with new evidence.

It was predictable that participants were working at different health care professions, so, the selected paper was based on general critical skills, consequently, inter professional relationship could be made by this virtual space, at the same time they could develop their reading professional reading during this process which could be considered as a continuing education at the same time in a friendly use approach. Obviously, improving professional knowledge and competence would be influential on quality of care, the goal of medical education.

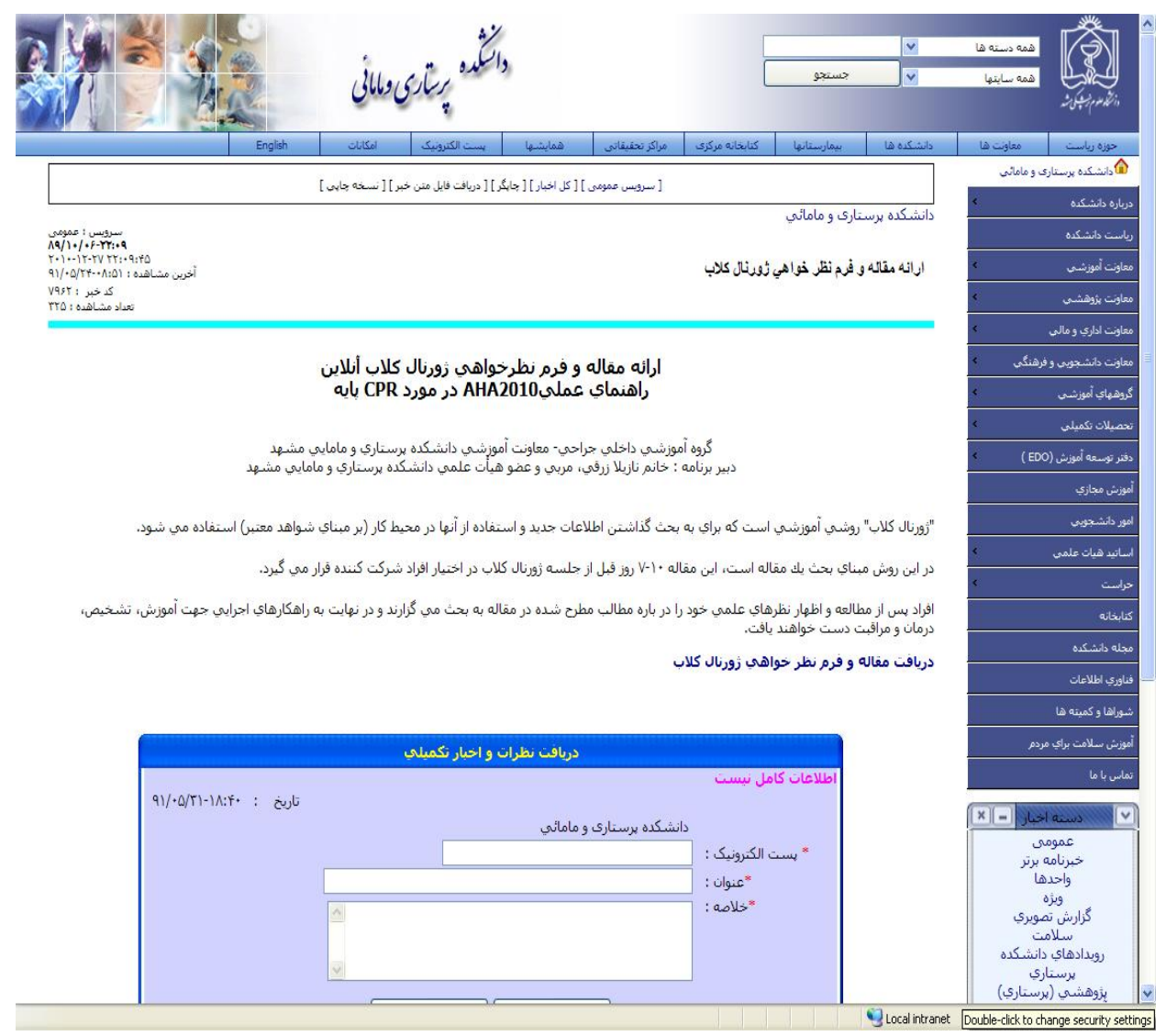

Figure 1.

The designed virtual spaces for electronic journal club: Early explanations and registration. 


\section{N. ZARGHI ET AL.}

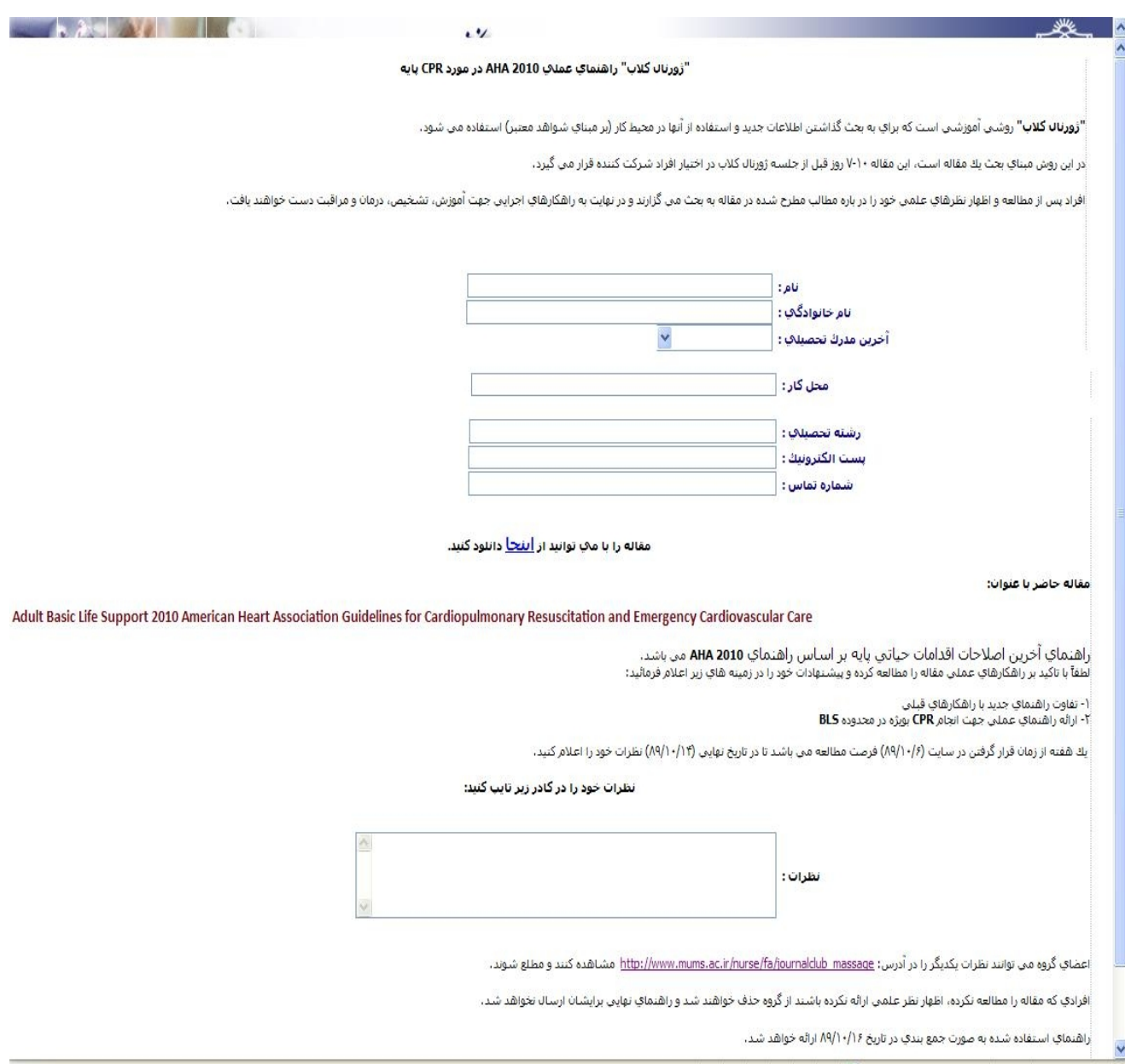

Figure 2.

The designed virtual spaces for electronic journal club: Receiving the paper and making comments.

\section{Results}

28 people registered and received the article, i.e. all of them read the instructions, aims and objectives of the online journal club and oriented with the process. Surprisingly, although participants included faculty members, students and university physicians were familiar with e-methods, no comment on the space was left. It means no journal club and no discussion was occurred but they read the article and got new information. Table 1 demonstrates the result of overall journal club objectives evaluation.

\section{Discussion}

As the body of medical literature continues to expand, healthcare teams especially physicians must develop the necessary skills to keep up with the vast amount of information. The journal club provides a forum to allow participants to remain up to date with the literature and is also teaches them the methods of critical evaluation. Those readers wishing to start a journal club or revive an existing one should begin by selecting a leader and defining the club goals. Formal evaluation will also provide an objective assessment of the knowledge gained by the housestaff through participation in journal club (Valentini \& Daniels, 1997).

At the start, journal clubs helped participants stay current with
Table 1.

Overall evaluation of objectives.

\begin{tabular}{lc}
\hline \multicolumn{1}{c}{ The aims and objectives of journal club } & $\begin{array}{c}\text { Overall } \\
\text { evaluation }\end{array}$ \\
\hline $\begin{array}{l}\text { Provide a bridge between research and practice and foster } \\
\text { an application of research to the clinical setting }\end{array}$ & + \\
Locate the best evidence on which to base practice & + \\
Help research awareness & + \\
Keep abreast with current research & + \\
Strengthen critical appraisal skills & - \\
Understand bio statistical and epidemiological methods & - \\
Increase confidence in dealing with evidence/research & + \\
Improving professional reading habits & + \\
Develop multidisciplinary relationships & \pm \\
Fulfill requirements for Continual Professional Development & \pm \\
$\begin{array}{l}\text { Maintain and improve professional knowledge and } \\
\text { competence which influence on quality of care }\end{array}$ & \pm \\
Develop local guidelines & + \\
\hline
\end{tabular}

Note: \pm : Doubtful; +: Achieved; -: Not achieved (main challenges). 
medical literature. Later, they improved acquisition of knowledge in clinical epidemiology, biostatistics, research design, and more recently in teaching critical appraisal skills. Journal clubs could develop critical thinking, reading habits and strengthening of collegial relationships. The journal club also has been advocated as a bridge between research and practice, hence facilitating better practice of evidence based medicine. Current internet technology has added another dimension to traditional journal club, where electronic even online discussions are the main stay of journal club. Many clinical disciplines report using journal clubs to train postgraduate trainees in relevant specialties. At present, benefits of a journal club exercise are reported not only in postgraduate programs, but also even documented at undergraduate level (Akhund \& Kadir, 2006).

As to the results, no journal club was conducted. A possible reason may be that the participants were in different Departments; participants were a mixed group of postgraduate students, nurses and physicians. Of the study limitations include the small sample size. Although the Mashhad University of Medical Sciences has one of the largest postgraduate programs and number of staffs in Iran, it was hypothesized that participants would discuss the article. The most optimistic explanation could be that the participants read the paper and got the new points, but they did not contribute to critical appraisal due to either lack of motivation or lack of knowledge about the way of participating in club despite of explanations. It seems, it was not considered as a priority for them. An alternative explanation would have been a significant interaction through shared duties and seminars among sites i.e. lack of time and they would likely have stopped attending. It was believed the best compromise was to be honest about the study. Nonetheless, the journal club in present study would not be conducted better than the others if it held. The results suggested that the journal club, as shown in this study, cannot be considered as an effective method for an electronic approach. In addition, the journal club did not describe an increased use of the literature, which corresponds to the earlier study findings. The format of a journal club is highly regarded as a way to teach skills for critical appraisal. However, this study corroborates the literature that journal clubs were not effective at the electronic approach especially in interactive offline versions. Perhaps participants have already acquired some skills, and further teaching produces diminishing returns. The effectiveness of journal clubs may root in other facets, such as improving collegiality and communicating new literature as well. Perhaps a wait-list group, may be feasible. Finally, it seems that the learning critical appraisal skills were promoted by the degree of personal importance to participants, and this factor is often in a form that affects on their evaluations.

\section{Conclusion}

Based on the results, it seems the main challenges were allocated to the objectives such as help research awareness, strengthen critical appraisal skills, understand bio statistical and epidemiological methods and increase confidence in dealing with evidence/research which need further education in non-virtual or even blended atmosphere. Some objectives were planned and supervised by leader/supervisor: provide a bridge between research and practice and foster an application of research to the clinical setting, locate the best evidence on which to base practice, improving professional reading habits, develop multidisciplinary relationships, fulfill requirements for Continual Professional Development and maintain and improve professional knowledge and competence which influence on quality of care. It should be said that evaluation of these objectives required specific methods and the results of this study just have presented overall evaluation. As the paper was not discussed electronically, the evaluation of the last three objectives, i.e. fulfill requirements for Continual Professional Development, maintain and improve professional knowledge and competence which influence on quality of care and develop local guidelines were in doubt.

As to the findings, it could be concluded that although participants were oriented with electronic methods and approaches, they did not participate in these sessions unless they gain some incentive points. Providing infrastructural factors such as allocating special time for participation and teaching them to run journal club, both educational and critical structure before participating in virtual ones and performing blended form of journal club before virtual ones could be helpful. Supervising clinical setting for applying them in practice is could be effective as well.

\section{Acknowledgements}

Special thanks to authorities of School of Nursing and Midwifery for their kind cooperation.

\section{REFERENCES}

Akhund, S., \& Kadir, M. M. (2006). Do community medicine residency trainees learn through journal club? An experience from a developing country. BMC Medical Education, 6, 43. doi:10.1186/1472-6920-6-43

Eval, J., Deenadayalan, Y., Grimmer-Somers, K., Prior, M., \& Kumar, S. (2008). How to run an effective journal club: A systematic review. Clinical Practice, 14, 898-911.

Gibbons, A. (2002). Organizing a successful journal club. BMJ Career Focus, 325, 137a.

Journal Club Support Service (2010). Leaflet prepared by NHS, Baldiston Healthcare Library. www.btuheks.nhs.uk/publications/journal_club_leaflet.pdf

Lindquist, R., Robert, R. C., \& Treat, D. (1990). A clinical practice journal club: Bridging the gap between research and practice. Focus on Critical Care, 17, 402-406.

Lizarondo, L., Kumar, S., \& Grimmer-Somers, K. (2010). Online journal clubs: An innovative approach to achieving evidence-based practice. Journal of Allied Health, 39, 17-22.

Linzer, M. (1987). The journal club and medical education: Over one hundred years of unrecorded history. Postgraduate Medical Journal, 63, 475-478. doi:10.1136/pgmj.63.740.475

Valentini, R. P., \& Daniels, S. R. (1997).The journal club. Postgraduate Medical Journal, 73, 81-85. doi:10.1136/pgmj.73.856.81 